\title{
Jet-disturbed molecular gas near the Seyfert 2 nucleus in M 51
}

\author{
S. Matsushita, S. Muller, and J. Lim
}

\begin{abstract}
Academia Sinica, Institute of Astronomy and Astrophysics, PO Box 23-141, Taipei 106, Taiwan, R.O.C. e-mail: satoki@asiaa.sinica.edu.tw
\end{abstract}

Received 28 December 2006 / Accepted 22 February 2007

\begin{abstract}
Context. Previous molecular gas observations at arcsecond-scale resolution of the Seyfert 2 galaxy M 51 suggest the presence of a dense circumnuclear rotating disk, which may be the reservoir for fueling the active nucleus and obscures it from direct view in the optical. However, our recent interferometric $\mathrm{CO}(3-2)$ observations show a hint of a velocity gradient perpendicular to the rotating disk, which suggests a more complex structure than previously thought.

Aims. To image the putative circumnuclear molecular gas disk at sub-arcsecond resolution to better understand both the spatial distribution and kinematics of the molecular gas.

Methods. We carried out $\mathrm{CO}(2-1)$ and $\mathrm{CO}(1-0)$ line observations of the nuclear region of $\mathrm{M} 51$ with the new A configuration of the IRAM Plateau de Bure Interferometer, yielding a spatial resolution lower than $15 \mathrm{pc}$.

Results. The high resolution images show no clear evidence of a disk, aligned nearly east-west and perpendicular to the radio jet axis, as suggested by previous observations, but show two separate features located on the eastern and western sides of the nucleus. The western feature shows an elongated structure along the jet and a good velocity correspondence with optical emission lines associated with the jet, suggesting that this feature is a jet-entrained gas. The eastern feature is elongated nearly east-west ending around the nucleus. A velocity gradient appears in the same direction with increasingly blueshifted velocities near the nucleus. This velocity gradient is in the opposite sense of that previously inferred for the putative circumnuclear disk. Possible explanations for the observed molecular gas distribution and kinematics are that a rotating gas disk disturbed by the jet, gas streaming toward the nucleus, or a ring with another smaller counter- or Keplarian-rotating gas disk inside.
\end{abstract}

Key words. galaxies: individual: M 51 - galaxies: individual: NGC 5194 - galaxies: ISM - galaxies: Seyfert

\section{Introduction}

Active Galactic Nuclei (AGNs) are believed to be powered by gas accretion. This gas is supplied from interstellar matter in host galaxies, and the gas may form rotationally-supported structures around the central supermassive black hole. If they are viewed close to edge-on, they may obscure the central activity from direct view. AGNs can be categorized as type 1 if seen face-on, and type 2 if seen edge-on; this explanation is known as a unified model (e.g. Antonucci \& Miller 1985). Indeed, a few hundred pc resolution molecular gas imaging toward the central regions of the Seyfert 2 galaxies NGC 1068 (Planesas et al. 1991; Jackson et al. 1993) and M 51 (Kohno et al. 1996) show strong peaks at the nuclei with velocity gradients perpendicular to radio jets, which suggest the existence of edge-on circumnuclear rotating disks. Recent $\sim 50$ pc resolution imaging studies toward NGC 1068 and the Seyfert 1 galaxy NGC 3227 support this view, showing more detailed structures, namely warped disks (Schinnerer et al. 2000a,b). However, observations toward a few low activity AGN galaxies with $<100 \mathrm{pc}$ resolution show lopsided, weak, or no molecular gas emission toward the nuclei (e.g. García-Burillo et al. 2003, 2005).

M 51 (NGC 5194) has also been observed in detail with molecular lines in the past, since it is one of the nearest $(7.1 \mathrm{Mpc}$; Takáts \& Vinkó 2006) Seyfert galaxies. A pair of radio jets emanates from the nucleus and narrow line regions (NLRs) are associated with the jet (e.g., Crane \& van der Hulst 1992; Grillmair et al. 1997; Bradley et al. 2004). Interferometric images in molecular gas show blueshifted emission on the eastern side of the Seyfert 2 nucleus, and redshifted gas on the western side (Kohno et al. 1996; Scoville et al. 1998). This shift is almost perpendicular to the jet axis, and the estimated column density is consistent with that estimated from X-ray absorption toward the nucleus, suggesting that the molecular gas can be a rotating disk and play an important role in obscuring the AGN. Interferometric $\mathrm{CO}(3-2)$ observations suggest a velocity gradient along the jet in addition to that perpendicular to the jet (Matsushita et al. 2004). These results imply more complicated features than a simple disk structure. We therefore performed sub-arcsecond resolution $\mathrm{CO}(2-1)$ and $\mathrm{CO}(1-0)$ imaging observations of the center of M51 to study the distribution and kinematics of the molecular gas around the AGN in more detail.

\section{Observation and data reduction}

We observed $\mathrm{CO}(2-1)$ and $\mathrm{CO}(1-0)$ simultaneously toward the nuclear region of M51 using the IRAM Plateau de Bure Interferometer. The array was in the new A configuration, whose maximum baseline length extends to $760 \mathrm{~m}$. Observations were carried out on February 4th, 2006. The system temperatures in DSB at $1 \mathrm{~mm}$ were in the range $200-700 \mathrm{~K}$, except for Antenna 6 , for which a new generation receiver gave system 


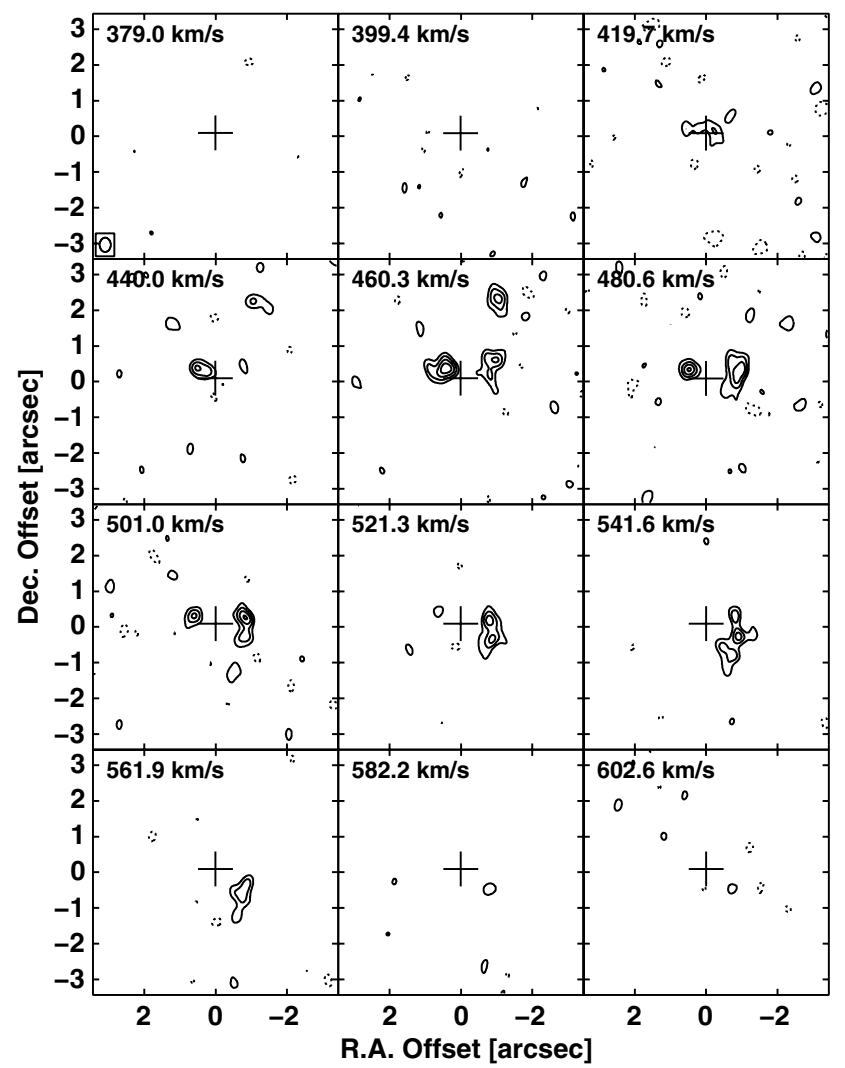

Fig. 1. Channel maps of the $\mathrm{CO}(2-1)$ line. The contour levels are $-3,3,5,7$, and $9 \sigma$, where $1 \sigma$ corresponds to $5.2 \mathrm{mJy} \mathrm{beam}^{-1}$ $(=0.96 \mathrm{~K})$. The cross in each map indicates the position of the $8.4 \mathrm{GHz}$ radio continuum peak position of $\mathrm{RA}=13^{\mathrm{h}} 29^{\mathrm{m}} 52^{\mathrm{s}} .7101$ and Dec $=$ 47 11'42'. 696 (Hagiwara et al. 2001; Bradley et al. 2004). The RA and Dec offsets are the offsets from the phase tracking center of RA = $13^{\mathrm{h}} 29^{\mathrm{m}} 52^{\mathrm{s}} .71$ and Dec $=47^{\circ} 11^{\prime} 42^{\prime \prime}$. 6 . The synthesized beam is shown at the bottom-left corner of the first channel map.

temperatures of $150-230 \mathrm{~K}$. Those in SSB at $3 \mathrm{~mm}$ were in the range $140-250 \mathrm{~K}$ for Antenna 6 , and $220-550 \mathrm{~K}$ for other antennas. Four of the correlators were configured to cover a $209 \mathrm{MHz}\left(272 \mathrm{~km} \mathrm{~s}^{-1}\right)$ bandwidth for the $\mathrm{CO}(2-1)$ line, and a $139 \mathrm{MHz}\left(362 \mathrm{~km} \mathrm{~s}^{-1}\right)$ bandwidth for the $\mathrm{CO}(1-0)$ line. The remaining four units of the correlator were configured to cover a $550 \mathrm{MHz}$ bandwidth for continuum observations and calibration. The strong quasar $0923+392$ was used for the bandpass calibration, and the quasars $1150+497$ and $1418+546$ were used for the phase and amplitude calibrations.

The data were calibrated using GILDAS, and were imaged using AIPS. The data were CLEANed with natural weighting, and the synthesized beam sizes are $0.40 \times 0$. . $31(14 \mathrm{pc} \times 11 \mathrm{pc})$ with a position angle $(\mathrm{PA})$ of $0^{\circ}$ and $0^{\prime} .85 \times 00^{\prime} .55(29 \mathrm{pc} \times 19 \mathrm{pc})$ with a PA of $13^{\circ}$ for $\mathrm{CO}(2-1)$ and $\mathrm{CO}(1-0)$ images, respectively. Figure 1 shows the channel maps of $\mathrm{CO}(2-1)$ emission with a $20.3 \mathrm{~km} \mathrm{~s}^{-1}$ velocity resolution. The channel maps of $\mathrm{CO}(1-0)$ emission show similar features to that of $\mathrm{CO}(2-1)$ emission with lower spatial resolution. Figure 2 shows integrated intensity and intensity weighted mean velocity maps of the $\mathrm{CO}(2-1)$ and $\mathrm{CO}(1-0)$ lines. The noise levels for continuum maps are $1.2 \mathrm{mJy}$ beam $^{-1}$ at $1.3 \mathrm{~mm}$ and $0.54 \mathrm{mJy}^{\mathrm{beam}}{ }^{-1}$ at $2.6 \mathrm{~mm}$, respectively. We did not detect any significant continuum emission at either frequency.
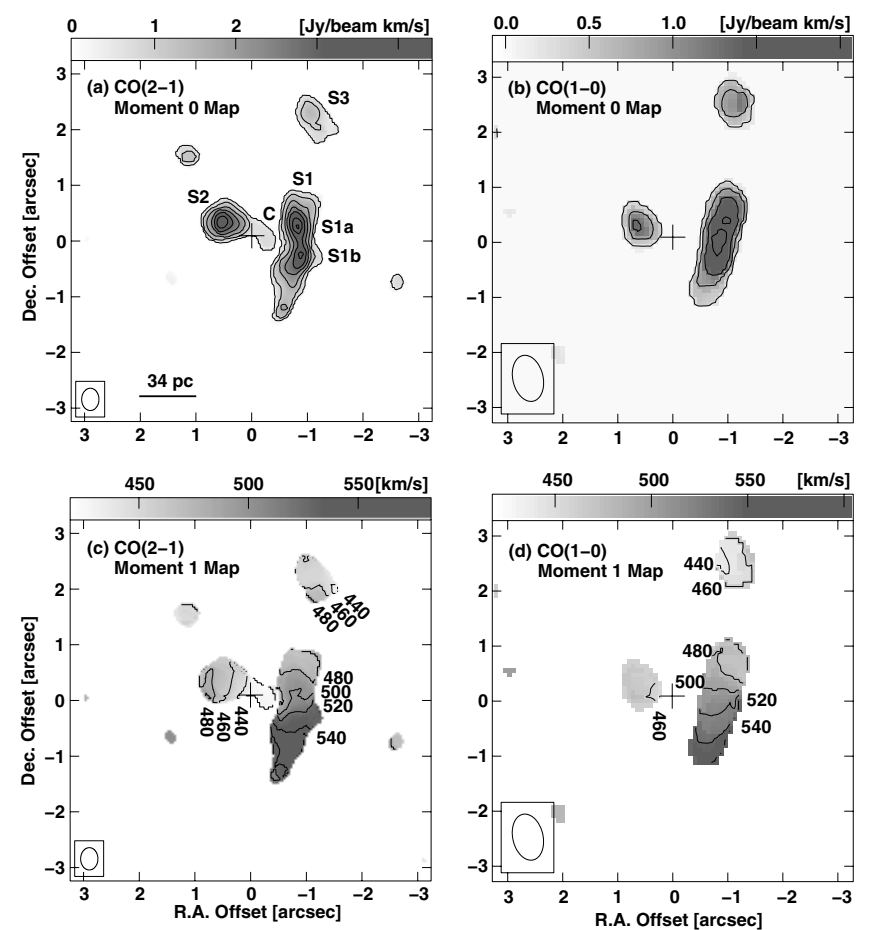

Fig. 2. Integrated intensity (moment 0 ) and intensity weighted velocity (moment 1 ) maps of the $\mathrm{CO}(2-1)$ and $\mathrm{CO}(1-0)$ lines. The synthesized beams are shown at the bottom-left corner of each image. The crosses and the reference positions of the RA and Dec offsets are the same as in Fig. 1. a) The $\mathrm{CO}(2-1)$ moment 0 image. The contour levels are $\left(1,3,5,7,9\right.$, and 11) $\times 0.334 \mathrm{Jy} \mathrm{beam}^{-1} \mathrm{~km} \mathrm{~s}^{-1}\left(=62.0 \mathrm{~K} \mathrm{~km} \mathrm{~s}^{-1}\right)$. b) The $\mathrm{CO}(1-0)$ moment 0 image. The contour levels are $(1,3,5$, and 7) $\times 0.257 \mathrm{Jy} \mathrm{beam}^{-1} \mathrm{~km} \mathrm{~s}^{-1}\left(=50.6 \mathrm{~K} \mathrm{~km} \mathrm{~s}^{-1}\right)$. c) The $\mathrm{CO}(2-1)$ moment 1 image. d) The $\mathrm{CO}(1-0)$ moment 1 image.

\section{Results}

Most of the $\mathrm{CO}(2-1)$ emission is detected within $\sim 1^{\prime \prime}(34 \mathrm{pc})$ of the center, and is located mainly on the eastern and western sides of the nucleus. There is also weak emission located $\sim 2$.' 7 northwest of the nucleus. The overall distribution and kinematics are consistent with past observations (Kohno et al. 1996; Scoville et al. 1998), if we degrade our image to lower angular resolution; a blueshifted feature with the average velocity of $\sim 460 \mathrm{~km} \mathrm{~s}^{-1}$ at the eastern side of the nucleus, and a redshifted feature with an average velocity of $\sim 500 \mathrm{~km} \mathrm{~s}^{-1}$ at the western side (Figs. 1, 2; see also Fig. 3b). We refer to these main structures with the same labels as in Scoville et al. (1998) (Fig. 2a).

Our higher resolution images, however, show more complicated structures and kinematics than the previous low angular resolution observations. Molecular gas on the western side of the nucleus, S1, is elongated in the north-south direction and separated into two main peaks (S1a and b). S1a is located 0.'9 (30 pc) northwest of the nucleus, and S1b is $11^{\prime \prime} 0(34 \mathrm{pc})$ to the southwest. On the eastern side of the nucleus, the molecular gas has an intensity peak 0.'6 (20 pc) to the northeast (labeled S2), which is located closer to the nucleus in projected distance than that of $\mathrm{S} 1 \mathrm{a} / \mathrm{b}$.

The feature S1 shows a clear velocity gradient along the north-south direction, which is shown in Fig. 2c and also in the position-velocity (PV) diagram (Fig. 3a). This gradient was previously suggested by the $\mathrm{CO}(3-2)$ data (Matsushita et al. 2004), but the magnitude of the velocity gradient is different. The computation of the magnitude of the velocity gradient is similar to that used for the $\mathrm{CO}(3-2)$ data. The fitting result indicates a 

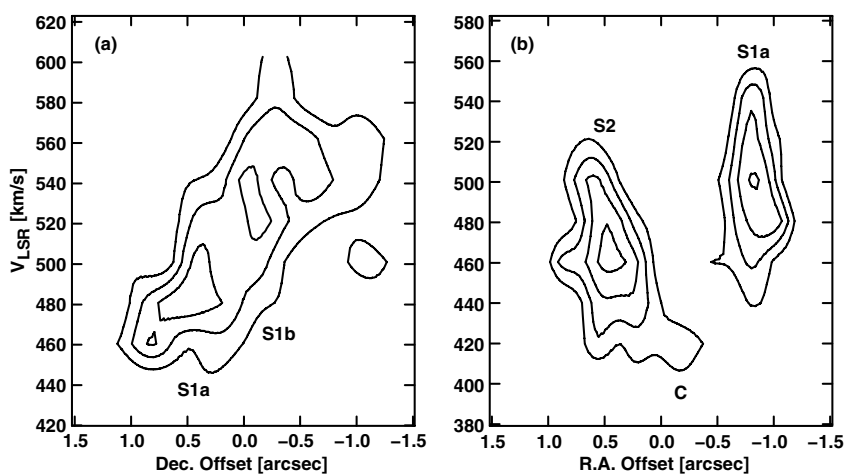

Fig. 3. Position-velocity (PV) diagrams of the $\mathrm{CO}(2-1)$ line. The contour levels are $3,5,7$, and $9 \sigma$, where $1 \sigma$ corresponds to $5.2 \mathrm{mJy}$ beam $^{-1}$ $(=0.96 \mathrm{~K})$. a) PV diagram along the north-south elongated S1 feature (PA of the cut is $103^{\circ}$ ). The positions for S1a and S1b are shown with labels. b) PV diagram along RA with the cut through the S1a, C, and $\mathrm{S} 2$ features (PA of the cut is $90^{\circ}$ ). The positions for $\mathrm{S} 1 \mathrm{a}, \mathrm{S} 2$, and $\mathrm{C}$ are shown with labels.

velocity gradient within $\mathrm{S} 1$ of $2.2 \pm 0.3 \mathrm{~km} \mathrm{~s}^{-1} \mathrm{pc}^{-1}$, which is larger than that reported previously, $0.77 \pm 0.01 \mathrm{~km} \mathrm{~s}^{-1} \mathrm{pc}^{-1}$ (the value has been modified by the different distance of the galaxy used). This difference is partially due to the larger beam size of the previous result; the $\mathrm{CO}(3-2)$ data set has a beam size of 3 .' $9 \times 11^{\prime \prime}$. 6 with a PA of $146^{\circ}$, and the velocities of S2/C and S3 contaminate that of $\mathrm{S} 1$.

The CO(1-0) maps show very similar molecular gas distribution and kinematics as those in $\mathrm{CO}(2-1)$ maps (Fig. 2b,d). Only the western emission was detected in previous observations (Aalto et al. 1999; Sakamoto et al. 1999), but our map clearly shows the emission from both side of the nucleus.

In addition to the previously known features, our $\mathrm{CO}(2-1)$ image also shows a weak emission near the nucleus with a structure elongated in the northeast-southwest direction (feature $\mathrm{C}$ in Fig. 2a). This structure could be a part of S2, since the velocity map (Fig. 2c) and the PV diagram (Fig. 3b) show a smooth velocity gradient, although most of the emission in $\mathrm{C}$ comes from only one velocity channel (419.7 $\mathrm{km} \mathrm{s}^{-1}$ map in Fig. 1). The velocity gradient between $\mathrm{S} 2$ and $\mathrm{C}$ is in an opposite sense to that previously seen with the lower angular resolution observations mentioned above. This structure is not detected in the $\mathrm{CO}(1-0)$ line, but a hint of a velocity gradient can be seen in Fig. 2 d.

The total $\mathrm{CO}(2-1)$ integrated intensity of $\mathrm{S} 1, \mathrm{~S} 2$, and $\mathrm{C}$ is $25.01 \mathrm{Jy} \mathrm{km} \mathrm{s}^{-1}$, and that of $\mathrm{S} 1$ and S2 in Scoville et al. (1998) is $33.44 \mathrm{Jy} \mathrm{km} \mathrm{s}^{-1}$, so that our data detected $75 \%$ of their intensity. Scoville et al. (1998) detected $\sim 50 \%$ and $20 \%$ of the single dish $\mathrm{CO}(2-1)$ flux in redshifted and blueshifted emission, respectively, so that our data recovered $\sim 25 \%$ of the single dish flux.

\section{Discussion}

\subsection{Jet-entrained molecular gas}

Our molecular gas data show a clear north-south velocity gradient within the feature $S 1$. We suggested from our previous study that this velocity gradient may be due to molecular gas entrainment by the radio jet (Matsushita et al. 2004). Here we revisit this possibility with higher spatial and velocity resolution data. Figure 4 shows our $\mathrm{CO}(2-1)$ image overlaid on the $6 \mathrm{~cm}$ radio continuum image (Crane \& van der Hulst 1992). The radio

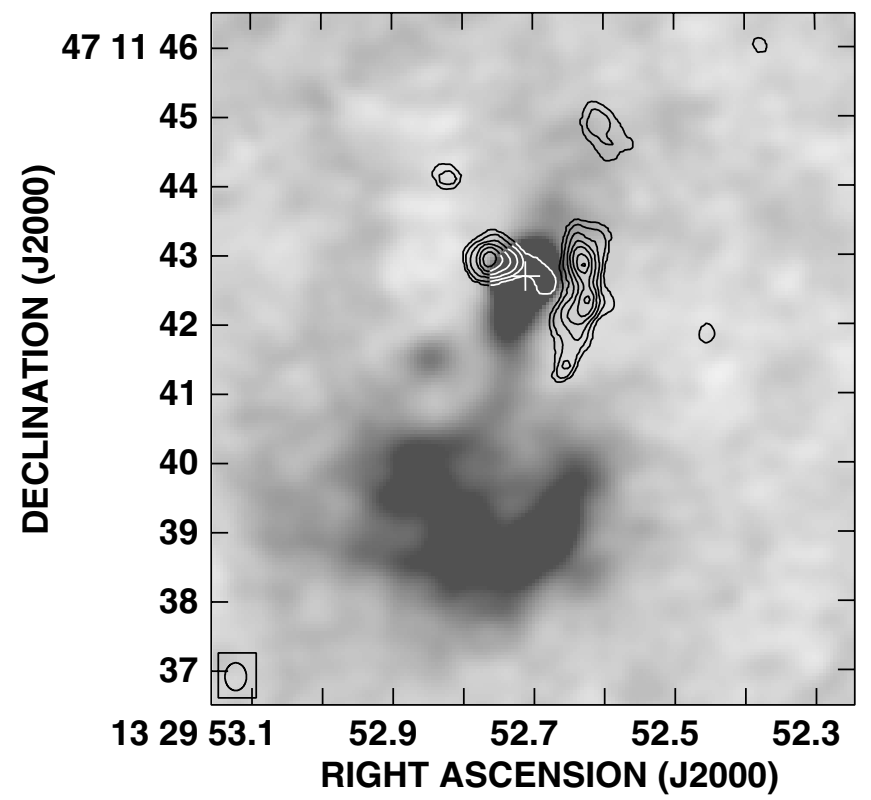

Fig. 4. The $\mathrm{CO}(2-1)$ integrated intensity image (contours) overlaid on the VLA $6 \mathrm{~cm}$ radio continuum image (greyscale; Crane \& van der Hulst 1992). The contour levels, the synthesized beam, and the cross are the same as in Fig. 1.

continuum image shows a compact radio core coincident with the nucleus, and the southern jet emanating from there (note that the northern jet is located outside our figure). The $\mathrm{CO}(2-1)$ map clearly shows that $\mathrm{S} 1$ is aligned almost parallel to the jet. In addition, Figs. 2 and 3 show that the velocity gradient in S1 is also almost parallel to the jet.

The velocity increases from $\sim 480 \mathrm{~km} \mathrm{~s}^{-1}$ at $\mathrm{S} 1 \mathrm{a}$ to $\sim 540 \mathrm{~km} \mathrm{~s}^{-1}$ south of $\mathrm{S} 1 \mathrm{~b}$. This increment is very similar to that observed in the NLR clouds along the radio jet; Bradley et al. (2004) measured the velocities and velocity dispersions of the clouds using the [O III] $\lambda 5007$ line, and showed that the velocity of the southern $\leqq 1^{\prime \prime}$ clouds from the nucleus are at $V_{\mathrm{LSR}} \sim 440-590 \mathrm{~km} \mathrm{~s}^{-1}$ and the velocity increases as the clouds move away from the nucleus (see Table 2 and Fig. 9 of their paper) ${ }^{1}$. This velocity range and increment are consistent with our data. Furthermore recent observations of $\mathrm{H}_{2} \mathrm{O}$ masers toward the nucleus also show a velocity gradient along the jet with the same sense as our results (Hagiwara 2007), in addtion to the good correspondance of the velocity range (Hagiwara et al. 2001; Hagiwara 2007; Matsushita et al. 2004). These results suggest that the molecular gas in S1 (and the NLR clouds and the $\mathrm{H}_{2} \mathrm{O}$ masers) is possibly entrained by the radio jet. These results also suggest that some of the material in NLRs is supplied from molecular gas close to AGNs.

Another example of jet-entrained neutral gas is found in the radio galaxy 3C 293 (Emont et al. 2005). The velocity of H I gas in absorption spectra toward the AGN matches that of ionized gas along kpc-scale radio jets. The spatial coincidence is not clear, since the spatial resolution of the $\mathrm{HI}$ data is lower $\left(25^{\prime \prime} .3 \times 11^{\prime \prime} .9\right)$ than that of the ionized gas data. Our result is therefore the first possible case of entrainment of molecular gas by a jet at the scale of ten pc.

1 We selected the clouds with a velocity dispersion of less than $100 \mathrm{~km} \mathrm{~s}^{-1}$; Clouds 3, 4, and 4a in Bradley et al. (2004). If we include all the clouds, the velocity is $\sim 440-690 \mathrm{~km} \mathrm{~s}^{-1}$ with a range of velocity dispersion of $\sim 25-331 \mathrm{~km} \mathrm{~s}^{-1}$; Clouds $2,3,3 \mathrm{a}, 4,4 \mathrm{a}$, and $4 \mathrm{~b}$. 
The better resolution of our new $\mathrm{CO}$ data allows us to revisit the values of the molecular gas mass, momentum, and energy of the entrained gas. We derive $6 \times 10^{5} M_{\odot}, 8 \times 10^{45} \mathrm{~g} \mathrm{~cm} \mathrm{~s}^{-1}$, and $3 \times 10^{52} \mathrm{erg}$ for these quantities. These values are about half of the previous values derived from the $\mathrm{CO}(3-2)$ data, mainly due to the larger beam, but the conclusion is similar; the energy of the entrained gas could be similar to that of the radio jet $\left(>6.9 \times 10^{51} \mathrm{erg}\right.$; Crane \& van der Hulst 1992), but the momentum is much larger than that of the jet $\left(2 \times 10^{41} \mathrm{~g} \mathrm{~cm} \mathrm{~s}^{-1}\right)$. One way to explain this discrepancy is through a continuous input of momentum from the jet (see Matsushita et al. 2004, for more detail discussions).

\subsection{Obscuring material around the Seyfert 2 nucleus}

The feature $\mathrm{C}$ is located in front of the Seyfert 2 nucleus, and the $\mathrm{CO}(2-1)$ intensity is about $62.0 \mathrm{~K} \mathrm{~km} \mathrm{~s}^{-1}$ (Fig. 2a). Hence the column density can be calculated as $6.2 \times 10^{21} \mathrm{~cm}^{-2}$ using a CO-to- $\mathrm{H}_{2}$ conversion factor of $1.0 \times 10^{20} \mathrm{~cm}^{-2}\left(\mathrm{~K} \mathrm{~km} \mathrm{~s}^{-1}\right)^{-1}$ (Matsushita et al. 2004) and assuming a $\mathrm{CO}(2-1) /(1-0)$ ratio of unity. This value is far lower than that derived from the X-ray absorption of $5.6 \times 10^{24} \mathrm{~cm}^{-2}$ (Fukazawa et al. 2001). As is mentioned in Sect. 3 , the missing flux of our data is $\sim 75 \%$. However, even if all of this missing flux contributes to obscuring the nuclear emission, this large column density difference cannot be explained. Changing the conversion factor or the ratio by an order of magnitude also cannot explain this large difference. One way to reconcile this disparity is to assume that $\mathrm{C}$ is not spatially resovled, in which case the computed column density is a lower limit. Alternatively, the obscuring material preferentially traced by higher- $J \mathrm{CO}$ lines or denser molecular gas tracers such as $\mathrm{HCN}$ may be involved. The $\mathrm{CO}(3-2)$ intensity in brightness temperature scale is $\sim 2$ times stronger than that of $\mathrm{CO}(1-0)$ (Matsushita et al. 2004), and the $\mathrm{HCN}(1-0)$ intensity is also relatively stronger $(\mathrm{HCN} / \mathrm{CO} \sim 0.4 ; \mathrm{Kohno}$ et al. 1996) than normal galaxies.

\subsection{Molecular gas at ten pc scale from the Seyfert nucleus}

Previous studies suggest that the blue shifted eastern feature S2 and the red shifted western feature $\mathrm{S} 1$ may be the outer part of a rotating disk as in the AGN unified model. However, our images show a more complicated nature, and no clear evidence of simple disk characteristics.

The simplest interpretation is that $\mathrm{S} 1$ and $\mathrm{S} 2 / \mathrm{C}$ are independent structures. Since $S 1$ is affected by the jet but $S 2 / C$ is not, $\mathrm{S} 1$ is expected to be located closer to the nucleus than $\mathrm{S} 2 / \mathrm{C}$, and the projection effect makes the position of $\mathrm{S} 2 / \mathrm{C}$ closer in our images. Alternatively, S2/C may be close to the nucleus, but the entrained gas has been already swept away or ionized by the jet. $\mathrm{S} 2 / \mathrm{C}$ has a velocity gradient, and therefore can be interpreted as a streaming gas, presumably infalling toward the nucleus, as is observed in the Galactic Center (Lo \& Claussen 1983; Ho et al. 1991).

$\mathrm{S} 1$ and $\mathrm{S} 2 / \mathrm{C}$ can also be interpreted as a rotating disk that is largely disturbed by the jet, and only a part remains. According to the velocity gradient along S2/C, the blueshfited gas is expected at $\mathrm{S} 1$, which is the opposite sense to the previous suggestion, but the gas shows no signs of it due to the jet entrainment. This is possible from the timescale point of view; under this interpretation, $\mathrm{S} 1$ should have a blueshifted rotation velocity of $\sim 380 \mathrm{~km} \mathrm{~s}^{-1}$ based on the velocity gradient in S2/C. S1 has a velocity $\sim 150 \mathrm{~km} \mathrm{~s}^{-1}$ higher than the expected rotational velocity, and we assume that this is the entrained velocity. In this case, it takes $2 \times 10^{5}$ years to be elongated along the jet by $\sim 1^{\prime \prime}$ or $34 \mathrm{pc}$. On the other hand, the rotation timescale at this radius is about $2 \times 10^{6}$ years, an order of magnitude longer timescale. The rotating disk can therefore be locally disturbed by the jet.

However, the above two explanations have difficulty in explaining optical images of the nucleus; the Hubble Space Telescope images show " $X$ " shaped dark lanes in front of the nucleus (Grillmair et al. 1997), suggesting the existence of a warped disk or two rings with one tilted far from another. An alternative explanation of the dark lanes is that, as previously proposed, there is a rotating edge-on ring with $\mathrm{S} 2$ as blueshifted gas and $\mathrm{S} 1 \mathrm{a}$ as redshifted gas. In this case, the feature $\mathrm{C}$ can be the counterpart of another dark lane, which runs northeastsouthwest, although $\mathrm{C}$ has to be a counter-rotating or Keplarian rotating disk to explain the opposite sense of the velocity gradient to that of the S1a/S2 (Sect. 3). This configuration explains the " $X$ " shape, but has a rather complicated configuration, and it is difficult to explain why the inner disk $\mathrm{C}$ is not disturbed by the jet.

We imaged the nuclear region of the Seyfert 2 galaxy M 51 at $\sim 10$ pc resolution, and we see no clear evidence of a circumnuclear rotating molecular gas disk as previously suggested. The molecular gas along the radio jet is most likely entrained by the jet. The explanations for other gas components are speculative, possibly involving a circumnuclear rotating disk or streaming gas.

Acknowledgements. We thank Arancha Castro-Carrizo and the IRAM staff for the new A configuration observations. We also thank the anonymous referee for helpful comments. IRAM is supported by INSU/CNRS (France), MPG (Germany) and IGN (Spain). This work is supported by the National Science Council (NSC) of Taiwan, NSC 95-2112-M-001-023.

\section{References}

Aalto, S., Hüttemeister, S., Scoville, N. Z., \& Thaddeus, P. 1999, ApJ, 522, 165 Antonucci, R. R. J., \& Miller, J. S. 1985, ApJ, 297, 621

Bradley, L. D., Kaiser, M. E., \& Baan, W. A. 2004, ApJ, 603, 463

Crane, P. C., \& van der Hulst, J. M. 1992, AJ, 103, 1146

Emonts, B. H. C., Morganti, R., Tadhunter, C. N., et al. 2005, MNRAS, 362, 931 Fukazawa, Y., Iyomoto, N., Kubota, A., Matsumoto, Y., \& Makishima, K. 2001, A\&A, 374, 73

García-Burillo, S., Combes, F., Hunt, L. K., et al. 2003, A\&A, 407, 485

García-Burillo, S., Combes, F., Schinnerer, E., Boone, F., \& Hunt, L. K. 2005, A\&A, 441, 1011

Grillmair, C. J., Faber, S. M., Lauer, T. R., et al. 1997, AJ, 113, 225

Hagiwara, Y. 2007, AJ, 133, 1176

Hagiwara, Y., Henkel, C., Menten, K. M., \& Nakai, N. 2001, ApJ, 560, L37

Ho, P. T. P., Ho, L. C., Szczepanski, J. C., Jackson, J. M., \& Armstrong, J. T. 1991, Nature, 350, 309

Jackson, J. M., Paglione, T. A. D., Ishizuki, S., \& Nguyen-Q-Rieu 1993, ApJ, 418, L13

Kohno, K., Kawabe, R., Tosaki, T., \& Okumura, S. K. 1996, ApJ, 461, L29

Lo, K. Y., \& Claussen, M. J. 1983, Nature, 306, 647

Matsushita, S., Sakamoto, K., Kuo, C.-Y., et al. 2004, ApJ, 616, L55

Planesas, P., Scoville, N., \& Myers, S. T. 1991, ApJ, 369, 364

Sakamoto, K., Okumura, S. K., Ishizuki, S., \& Scoville, N. Z. 1999, ApJS, 124, 403

Schinnerer, E., Eckart, A., \& Tacconi, L. J. 2000a, ApJ, 533, 826

Schinnerer, E., Eckart, A., Tacconi, L. J., Genzel, R., \& Downes, D. 2000b, ApJ, 533,850

Scoville, N. Z., Yun, M. S., Armus, L., \& Ford, H. 1998, ApJ, 493, L63

Takáts, K., \& Vinkó, J. 2006, MNRAS, 372, 1735 